\title{
Nuclear forces from EFT: Recent developments
}

\author{
H. Krebs ${ }^{1,2, a}$, E. Epelbaum ${ }^{2,1}$, and U.-G. Meißner ${ }^{1,2,3}$ \\ 1 Helmholtz-Institut für Strahlen- und Kernphysik (Theorie) and Bethe Center for Theoretical Physics, \\ Universität Bonn, D-53115 Bonn, Germany \\ 2 Forschungszentrum Jülich, Institut für Kernphysik (IKP-3) and Jülich Center for Hadron Physics, \\ D-52425 Jülich, Germany \\ 3 Forschungszentrum Jülich, Institut für Kernphysik (IKP-3), Institite for Advanced Simulation (IAS-4) \\ and Jülich Center for Hadron Physics, D-52425 Jülich, Germany
}

\begin{abstract}
Nuclear forces are considered based on chiral perturbation theory with and without explicit $\Delta$-isobar degrees of freedom. We discuss the subleading corrections to chiral three-nucleon forces in the $\Delta$-less formalism which contain no additional free parameters. In the formalism with explicit $\Delta$-isobar we present the complete next-to-next-to-leading order analysis of isospin-conserving and next-to-leading order analysis of isospinviolating nuclear forces. The perturbative expansion of nuclear forces in the $\Delta$-full case is shown to have much better convergence compared with the $\Delta$-less theory where the $\Delta$-resonance is integrated out and is encoded in certain low-energy constants.
\end{abstract}

\section{Nuclear forces in chiral effective field theory}

One of the most important questions in nuclear physics is how the nucleons as the constituents of nuclei interact with each other. Already 1935 Yukawa made an attempt to explain the nature of the nucleon-nucleon interaction by a virtual meson exchange between nucleons [1]. Later the discovery of pions and heavier mesons laid the foundation for the development of highly sophisticated phenomenological models for nuclear forces which were motivated by the original idea of Yukawa. In the two-nucleon sector they give a perfect description of the experimental data at the cost of often more than 40 parameters fixed from the fit to data. In the three-nucleon sector it is probably not feasible to follow a similar phenomenological path. The rich spin-isospin structure allows much more possibilities to parameterize the three-nucleon force leading to much more unknown constants which can hardly be fixed from the experimental input.

In order to improve our understanding of nuclear forces we need to learn how they are connected to the underlying theory of strong interactions, Quantum Chromo Dynamics (QCD). In the above mentioned phenomenological models this connection is obviously missing. From the QCD point of view, nuclear forces are given as residual interaction between hadrons described by quark-gluon dynamics. However, in the low-energy sector far below the chiral-symmetry-breaking scale $\Lambda_{\chi} \sim 1 \mathrm{GeV}$, quarks and gluons are not the most efficient degrees of freedom for the

\footnotetext{
a e-mail: hkrebs@itkp.uni-bonn.de
}

description of nuclear processes. Nuclear forces in this region are largely driven by chiral Goldstone boson dynamics which appears due to spontaneous and explicit breaking of chiral symmetry in QCD. In the SU(2) scenario the Goldstone bosons can be interpreted as pions which get their mass due to the explicit chiral symmetry breaking by the small up and down quark masses. Since pions are Goldstone bosons, their interactions vanish with vanishing fourmomenta. This fact allows us to construct an effective field theory (EFT) with nucleons and pions as degrees of freedom and the same underlying symmetries of QCD in form of a perturbative expansion in low external momenta and pion mass divided by the hard scale $\Lambda_{\chi} \sim 1 \mathrm{GeV}[2,3]$. In this systematic, order-by-order improvable approach called chiral perturbation theory (ChPT) we get both a direct connections to QCD and a description in efficient hadronic (rather than quark) degrees of freedom by construction.

In the pure meson and one-nucleon sectors, ChPT has been used to calculate various processes like form factors, pion-nucleon, Compton scattering etc. (see [4] for a recent review). In the two and more nucleon sector an additional problem appears which does not allow to use ChPT as just a perturbation theory. Almost two decades ago we learned from the seminal paper of Weinberg [5] that diagrams with two (or more) nucleon cuts violate the power counting of ChPT. As a solution to this problem he suggested to define an effective potential which per construction should not have any two (or more) nucleon cuts and thus can be calculated perturbatively to a desired order by using chiral EFT. In order to describe a given nuclear process we need to solve Lippman-Schwinger, Faddeev or 
Faddeev-Jakubowsky equations numerically (with the previously constructed effective potential as input) in the two-, three-, four- and more-nucleon sector, respectively.

This path has been followed in the last two decades by several groups. The chiral effective nucleon-nucleon (NN) potential has been constructed up to next-to-next-to-nextto-leading-order $\left(\mathrm{N}^{3} \mathrm{LO}\right)$. At this order 24 unknown lowenergy-constants (LECs) coming from short-range NN contact interactions have been fitted to the proton-neutron scattering phase shifts obtained from the Nijmegen partial wave analysis (PWA) [6,7]. At $\mathrm{N}^{3} \mathrm{LO}$ the chiral effective NN potential describes the Nijmegen PWA phases almost as good as other phenomenological potentials. The three-body forces have been analyzed numerically only up to $\mathrm{N}^{2} \mathrm{LO}$. At this order there appear two additional short-range LECs which have been fitted e.g. to the binding energy of ${ }^{3} \mathrm{H}$ and $\mathrm{S}$ wave doublet neutron-deuteron scattering length. At $\mathrm{N}^{2} \mathrm{LO}$ the description of three-body observables is in most cases satisfactory. The results for the differential cross section of elastic neutron-deuteron scattering e.g. are in good agreement with experimental data. On the other hand there are some observables which are rather poorly described at this order even at low energies. One example is the so-called symmetric space-star configuration in neutron-deuteron break up reaction. In this configuration, the plane in the CMS spanned by the outgoing nucleons is perpendicular to the beam axis, and the angles between the nucleons are $120^{\circ}$. At $E_{\mathrm{lab}}=15 \mathrm{MeV}$ theoretical calculations based on both phenomenological and chiral nuclear forces are unable to describe the data (see recent reviews $[9,10]$ for extensive discussion). There is, however, hope of improvement once $\mathrm{N}^{3} \mathrm{LO}$ three-body forces are included.

\section{$2 \mathrm{~N}^{3} \mathrm{LO}$ chiral three-nucleon force}

An interesting point about the $\mathrm{N}^{3} \mathrm{LO}$ contributions to chiral three-nucleon forces is the absence of additional LECs at that order. Their potential appearance is just prevented by the underlying symmetries of QCD. The rich spin-isospin structure of the $\mathrm{N}^{3} \mathrm{LO}$ contributions makes them also promising to resolve the discrepancies in the description of some until now poorly described three-nucleon observables.

The $\mathrm{N}^{3} \mathrm{LO}$ contributions can be divided into two parts. The first part is given by long-range contributions which include following contributions:

- Two-pion exchange ( $2 \pi$ ) graphs (see graph (a) in Fig. 1) which have been considered by Ishikawa and

Robilotta [12] using the so-called infrared regularization method and by our group [13] in the framework of unitary transformations combined with Heavy Baryon formalism.

- Two-pion-one-pion exchange $(2 \pi-1 \pi)$ graphs which are visualized by graph (b) in Fig. 1.

- Three-pion exchange, so-called ring diagrams, which are visualized by graph (c) in Fig. 1.

Analytic expressions of all these contributions can be found in [13]. The second part is given by shorter-range contributions which is visualized by graphs (d) and (e) in Fig.1 and corresponding $1 / m$ corrections (with $m$ the nucleon mass). Their construction has been by now finished and will be published elsewhere. It is important to note that the construction of the $\mathrm{N}^{3} \mathrm{LO}$ forces is unique only modulo unitary transformations. In the static limit, however, the natural choice of unitary transformation is entirely dictated by renormalizability requirement of the three-nucleon forces [14]. One can even speculate that in general an effective potential in the static limit is unique once we additionally require its renormalizability.

\section{Chiral EFT with $\Delta$-isobar degrees of freedom}

In the standard chiral EFT discussed so far only pions and nucleons are treated as dynamical degrees of freedom. All other resonances like e.g. $\Delta$ or $\rho$ are integrated out and their contributions are encoded in the LECs. In this scheme pion four-momenta $Q$ and mass $M_{\pi}$ are treated as a soft scale and the mass difference $\Delta=m_{\Delta}-m_{N}$ between the lightest baryon $\Delta$-resonance and nucleon as a hard scale which is assumed to be of the same size as the chiral symmetry breaking scale $\Lambda_{\chi} \sim 1 \mathrm{GeV}$ :

$$
Q \sim M_{\pi} \ll \Delta=293 \mathrm{MeV} .
$$

One can still argue that scales of the order of $\sim 300 \mathrm{MeV}$ can be treated as a soft scale. In this case one has to introduce the $\Delta$ degrees of freedom explicitely into the theory and enlarge the set of expansion parameters by the deltanucleon mass difference $\Delta / \Lambda_{\chi}$ :

$$
Q \sim M_{\pi} \sim \Delta \ll \Lambda_{\chi} .
$$

This expansion is known in the literature as the small scale expansion (SSE) [15]. Integrating out of $\Delta$ degrees of freedom leads often to an enlargement of the values of the LECs. This e.g. happens with the $\pi \pi N N$ LECs $c_{3}$ and $c_{4}$ which are saturated by the $\Delta$-isobar and are known to be unnaturally large. In the theory with explicit $\Delta$, however, they have natural size. Therefore the unnatural enlargement of some $c_{i}$ 's in the delta-less theory can be explained by large $\Delta$-contributions which get absorbed by the $c_{i}$.

The appearance of unnaturally large LECs in an EFT can spoil its convergence. This can be seen in the chiral effective potential: subleading-order contributions to chiral $2 \pi$-exchange potential in the $\Delta$-less theory appears to be larger than the leading one. From this point of view, the explicit inclusion of $\Delta$-isobar is well motivated: One can expect the LECs to be of natural size and the SSE of chiral nuclear forces to possess a natural convergence.

\section{Nuclear forces with explicit $\Delta$-isobar}

The consideration of chiral nuclear forces with explicit $\Delta$ isobar started more than one decade ago. Ordonez et al. [16] and Kaiser et al. [17] worked out the leading $\Delta$-resonance contributions to the chiral nuclear forces. They have shown 


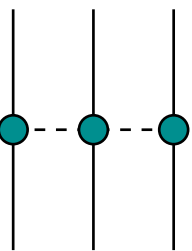

(a)

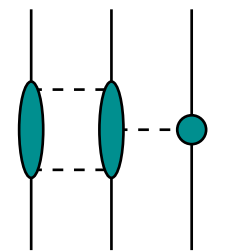

(b)

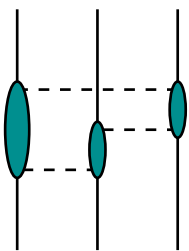

(c)

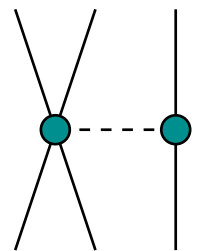

(d)

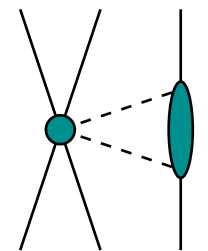

(e)

Fig. 1. Various topologies that appear in the three-nucleon force at $\mathrm{N}^{3} \mathrm{LO}$. Solid and dashed lines represent nucleons and pions, respectively. Shaded blobs are the corresponding amplitudes. The long-range part of the three-nucleon force considered in this paper consists of (a) $2 \pi$ exchange graphs, (b) $2 \pi-1 \pi$ diagrams and the ring diagrams (c). The topologies (d) and (e) involve four-nucleon contact operators and are of shorter range.

that the large $\mathrm{N}^{2} \mathrm{LO}$ contributions to the $2 \pi$-exchange potential in the standard chiral EFT are shifted to NLO such that the dominant contribution to the nuclear forces appear already at NLO in the $\Delta$-full scenario. In the standard chiral EFT three-nucleon forces start to contribute at $\mathrm{N}^{2} \mathrm{LO}$. This changes in a $\Delta$-full theory where the first three-nucleon contribution starts already at NLO and is given by the well known Fujita-Miyazawa force [19,20]. Recently we calculated the subleading $\Delta$-resonance effects [18] which contribute to $\mathrm{N}^{2} \mathrm{LO}$ chiral nuclear forces. An interesting point is that at $\mathrm{N}^{2} \mathrm{LO}$ there are no corrections to the three-nucleon forces coming from $\Delta$-isobar excitations: all possible NNN $\Delta$ contact interactions are forbidden by the Pauli exclusion principle. The forces constructed out of building blocks with that contact interaction disappear after antisymmetrization. So the only non-zero $\Delta$-resonance contribution to the three-nucleon forces upto $\mathrm{N}^{2} \mathrm{LO}$ is given by the FujitaMiyazawa force [21].

In order to discuss numerical results for the $\Delta$-full forces upto $\mathrm{N}^{2} \mathrm{LO}$ we need to fix all the unknown constants which appear at this order. These are leading and subleading combination of the pion-nucleon-delta coupling constants $h_{A}$ and $b_{3}+b_{8}$, respectively, and $c_{i}, i=1,2,3,4$, from $\pi N$ sector. We fixed them in two different fits to the $S$ - and P-wave threshold parameters of pion-nucleon scattering calculated upto second order in SSE. The diagrams contributing to this order are shown in Fig. 2. In the first fit we take large $N_{c}$ value for

$$
h_{A}=\frac{3 g_{A}}{2 \sqrt{2}} \simeq 1.34,
$$

where $g_{A}=1.27$ is the nucleon axial vector coupling, and fitted all other constants to various threshold parameter. In the second fit we used $h_{A}=1.05$ which has been extracted from the Heavy-Baryon $\Delta$-width analysis in the static limit [15] and is consistent with quark model relation.

$$
h_{A}=\frac{6 g_{A}}{5 \sqrt{2}} \simeq 1.07 \text {. }
$$

Numerical values for various LECs determined in this way are given in Table 1 . Note that the constants $c_{3}$ and $c_{4}$ are strongly reduced in the $\Delta$-full theory which is consistent with our previous considerations. With these constants we found for all effective NN potentials much better convergence compared with the potentials in the delta-less theory (see [18] for an extensive discussion). The same behavior can be seen in peripheral partial waves (see e.g. ${ }^{3} F_{3^{-}}$ and ${ }^{3} F_{4}$-partial waves within $\Delta$-full and $\Delta$-less theories in comparison with the Nijmegen and Virginia Tech PWA in Fig. 3). Note that although the convergence in the $\Delta$-full theory is much better than in the $\Delta$-less one, the overall $\mathrm{N}^{2} \mathrm{LO}$ results in both formulations are very similar.

\subsection{Leading isospin-breaking $\Delta$-isobar contributions}

To estimate the size of isospin-breaking effects we also studied isospin-breaking contributions to chiral NN forces within the $\Delta$-full theory up to next-to-leading order. In this presentation we concentrate only on charge symmetry breaking (CSB) contributions to nuclear forces. For the full discussion see [23] and the forthcoming publication [24].

The leading CSB contributions to the nuclear force in the $\Delta$-full theory are proportional to the nucleon- and $\Delta$ mass splittings. At leading order there are electromagnetic and strong isospin-breaking contributions to the $\Delta$-mass splittings. While both of them contribute to the equidistant splitting $\delta m_{\triangle}^{1}$ in the $\Delta$ quartet, the non-equidistant splitting $\delta m_{\Delta}^{2}$ is of a pure electromagnetic origin. To estimate the values of the $\Delta$-mass splittings we proceeded in two ways. In the first fit we used the most recent Particle Data Group values for

$m_{\Delta^{++}}=1230.80 \pm 0.30 \mathrm{MeV}, \quad m_{\Delta^{0}}=1233.45 \pm 0.35 \mathrm{MeV}$

together with the average mass $m_{\Delta}=1233 \mathrm{MeV}$. With this input we get for the $\Delta$-mass splittings

$$
\delta m_{\Delta}^{1}=-5.3 \pm 2.0 \mathrm{MeV}, \quad \delta m_{\Delta}^{2}=-1.7 \pm 2.7 \mathrm{MeV} .
$$

Alternatively, instead of using $m_{\Delta}=1233 \mathrm{MeV}$, we employed the quark model relation [25]

$$
m_{\Delta^{+}}-m_{\Delta^{0}}=\delta m_{N},
$$

where $\delta m_{N}$ is the nucleon mass splitting. The results for the $\Delta$-mass splittings determined in this way appear to be 

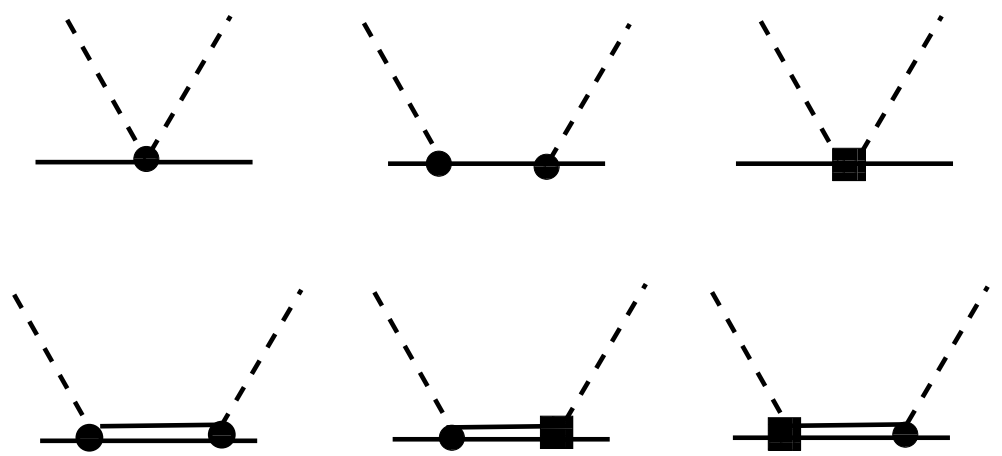

Fig. 2. Tree graphs for pion-nucleon scattering at NLO. The filled circles/squares denote leading/subleading vertices. Crossed graphs are not shown.

\begin{tabular}{||c||c|c|c||c||}
\hline \hline LECs & $Q^{2}$, no $\Delta$ & $Q^{2}$, fit 1 & $Q^{2}$, fit 2 & $Q^{3}$ no $\Delta[22]$, fit 2 \\
\hline \hline$c_{1}$ & -0.57 & -0.57 & -0.57 & $-1.42 \pm 0.03$ \\
$c_{2}$ & 2.84 & -0.25 & 0.83 & $3.13 \pm 0.04$ \\
$c_{3}$ & -3.87 & -0.79 & -1.87 & $-5.58 \pm 0.01$ \\
$c_{4}$ & 2.89 & 1.33 & 1.87 & $3.50 \pm 0.01$ \\
$h_{A}$ & - & $1.34^{\star}$ & $1.05^{\star}$ & - \\
$b_{3}+b_{8}$ & - & 1.40 & 2.95 & - \\
\hline \hline
\end{tabular}

Table 1. Determinations of the LECs from S- and P-wave threshold parameters in $\pi N$ scattering based on the $Q^{2}$ fits with and without explicit $\Delta$ 's. LECs used as input are marked by the star. Also shown are the values determined in Ref. [22] from fit 2 at $Q^{3}$ without explicit $\Delta$ 's (the errors given are purely statistical and do not reflect the true uncertainty of the LECs). The LECs $c_{i}$ and $b_{3}+b_{8}$ are given in $\mathrm{GeV}^{-1}$.
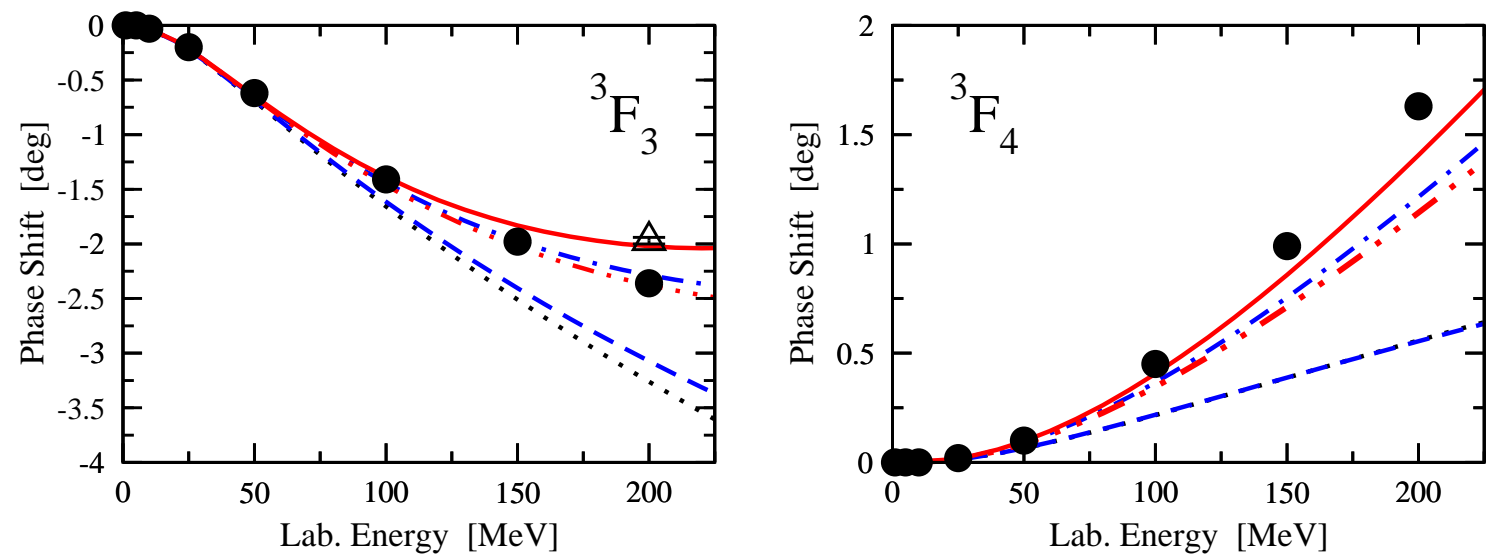

Fig. 3. ${ }^{3} F_{3}$ - and ${ }^{3} F_{4}$-wave NN phase shifts. The dotted curve is the LO prediction (i.e. based on the pure one-pion-exchange potential). Dashed double-dotted (dashed) and solid (dashed-dotted) lines show the NLO and $\mathrm{N}^{2}$ LO results with (without) the explicit $\Delta$-contributions. The filled circles (open triangles) depict the results from the Nijmegen multi-energy PWA [6,7] (Virginia Tech singleenergy PWA [8])

consistent with the first determination:

$$
\delta m_{\Delta}^{1}=-3.9 \mathrm{MeV}, \quad \delta m_{\Delta}^{2}=0.3 \pm 0.3 \mathrm{MeV} .
$$

Having determined the values for the $\Delta$-mass splittings we studied the CSB contributions to the nuclear forces. In Fig. 4 we show CSB two-pion-exchange contributions to the two-nucleon potential

$$
V=\left(\tau_{1}^{3}+\tau_{2}^{3}\right)\left(V_{C}^{I I I}+V_{S}^{I I I} \sigma_{1} \cdot \sigma_{2}+V_{T}^{I I I} \sigma_{1} \cdot \boldsymbol{q} \sigma_{2} \cdot \boldsymbol{q}+\ldots\right)
$$

in configuration space. The contributions due to the nucleonmass splitting $\delta m_{N}$ for the potentials $V_{S, T}^{I I I}$ appear to be similar in the $\Delta$-less and $\Delta$-full theories. In the central potential $V_{C}^{I I I}$, however, we see sizeable differences. In all potentials we observe strong cancellations between the $\delta m_{N^{-}}$ and $\delta m_{\Delta}^{1}$-contributions which lead to significantly weaker $V_{C, S, T}^{I I I}$ potentials in the $\Delta$-full theory. 

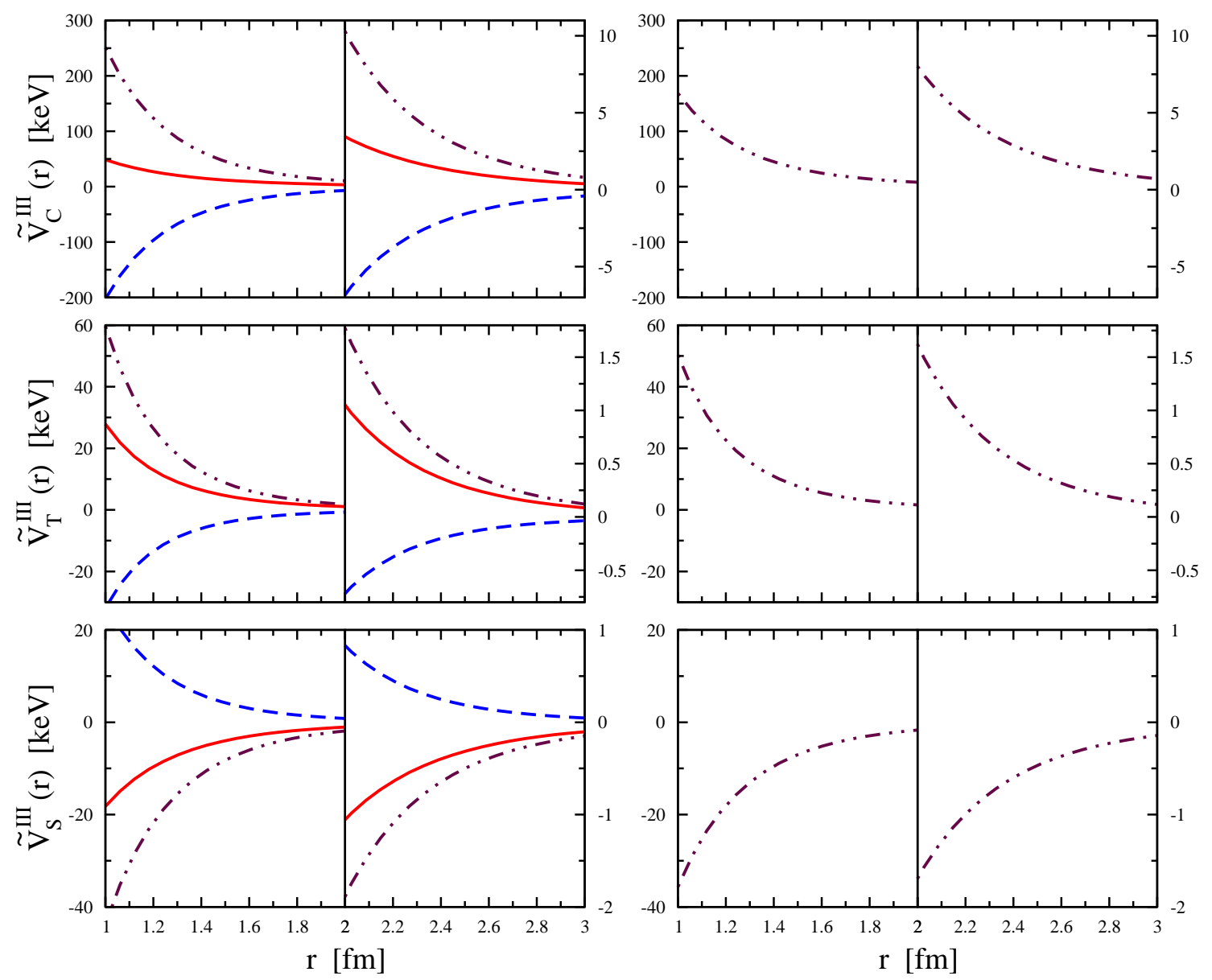

Fig. 4. CSB two-pion exchange potential. The left (right) panel shows the results obtained at leading order in chiral EFT with explicit $\Delta$-resonances (at subleading order in chiral EFT without explicit $\Delta$ degrees of freedom). The dashed and dashed-double-dotted lines depict the contributions due to the $\Delta$ - and nucleon-mass differences $\delta m_{\Delta}^{1}$ and $\delta m_{N}$, respectively, while the solid lines give the total result.

\subsection{Subleading isospin-breaking $\Delta$-isobar contributions}

In order to study the convergence of the isospin-breaking nuclear forces within SSE we need to calculate them at least up to next-to-leading order. We concentrate here again on the two-pion-exchange part of CSB forces and refer to forthcoming publication [24] for the full discussion. At NLO there appear three unknown constants: one of them is $\beta$ which is a combination of the LECs accompanying the leading isospin-breaking $\pi N N$ vertex [11]. Two other unknown constants which we call $\beta_{A}^{1}$ and $\beta_{A}^{2}$ are given by a particular combination of the LECs accompanying the leading isospin-breaking $\pi N \Delta$ vertex [24]. For our current considerations we set all the three constants to zero. Similar to LO there are two contributions to CSB forces at NLO which are proportional to nucleon- and $\Delta$-mass splittings $\delta m$ and $\delta m_{\Delta}^{1}$, respectively.

In Fig. 5 we show individual contributions to CSBforces at NLO (right panel) compared with the corresponding forces at leading order (left panel). NLO corrections to CSB-forces appear to be of a natural size. In all the potentials both at LO and at NLO we observe strong cancellations between the contributions proportional to $\delta m$ and $\delta m_{4}^{1}$. In all CSB-forces at NLO, however, one can see that $\delta m$-contributions are almost by factor 2 larger than $\delta m_{\Lambda^{1}}^{1}$ contributions. This leads to stronger $V_{C, S, T}^{I I I}$ potentials even in the $\Delta$-full theory. The entire effect which leads to weaker CSB forces at leading order in SSE and which can be interpreted as higher order effects from the resonance saturation point of view in the $\Delta$-less theory is completely compensated at NLO in SSE. This can be clearly seen from Fig. 6 where we show the results for CSB-forces up to NLO both in the $\Delta$-full (left panel) and $\Delta$-less theory (right panel). Similar to the isospin-conserving case one can see that the overall results in both theories with and without explicit $\Delta$ degrees of freedom are remarkably close. Only the convergence of the nuclear forces is improved once the $\Delta$ is explicitely taken into account.

\subsection{Summary}

We discussed the long- and shorter-range $\mathrm{N}^{3} \mathrm{LO}$ contributions to chiral three-nucleon forces. No additional free parameters do appear at this order. There are five different topology classes which contribute to the forces. Three of them describe long-range contributions which consti- 

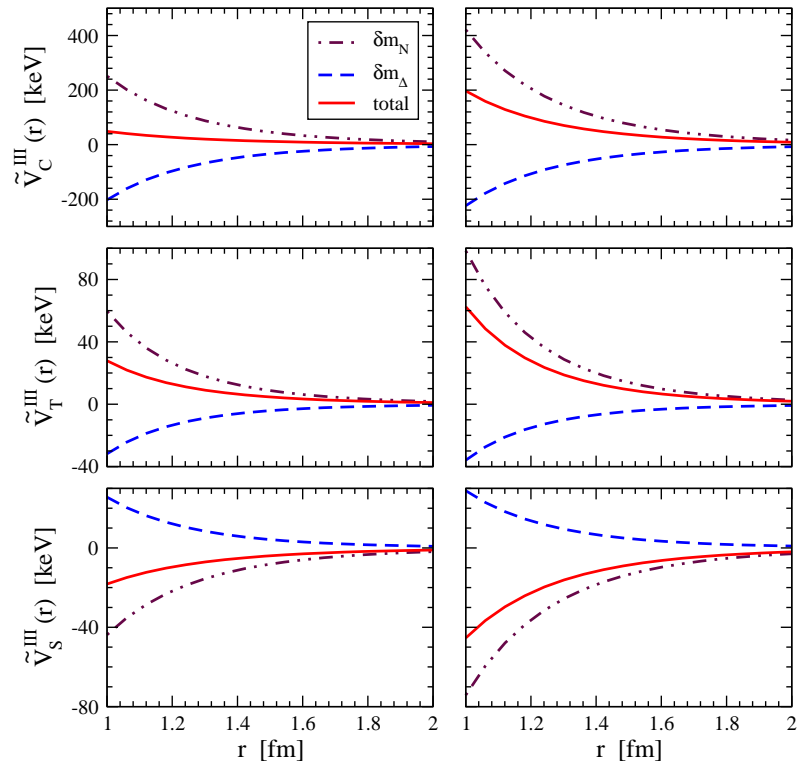

Fig. 5. CSB two-pion exchange potential. The left (right) panel shows the results obtained at leading order (at subleading order) in chiral EFT with explicit $\Delta$-resonances. The dashed and dasheddouble-dotted lines depict the contributions due to the $\Delta$ - and nucleon-mass differences $\delta m_{4}^{1}$ and $\delta m_{N}$, respectively, while the solid lines give the total result.
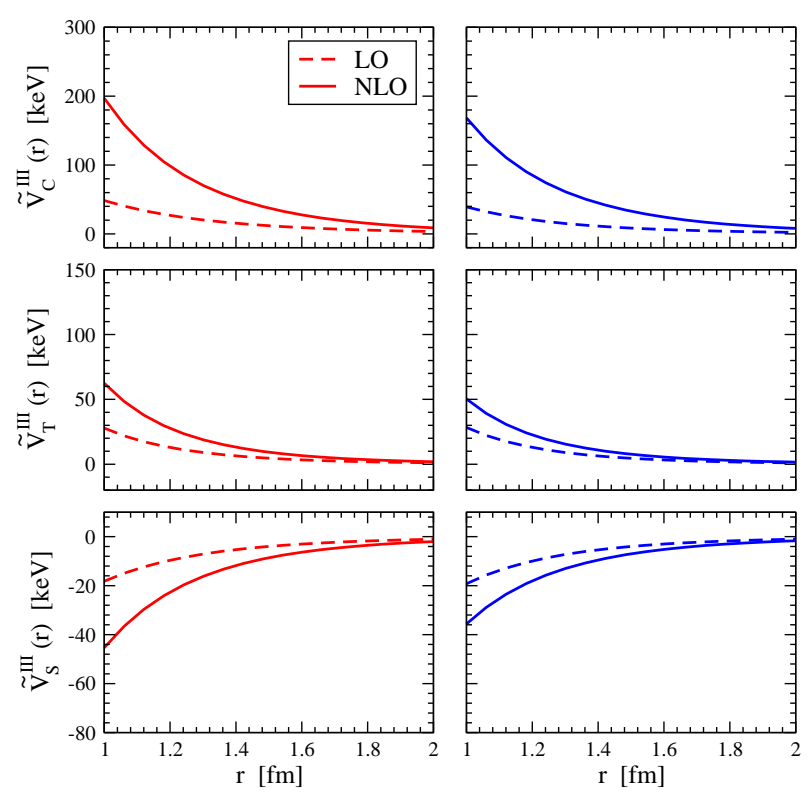

Fig. 6. CSB two-pion exchange potential. The left (right) panel shows the results obtained up to NLO in chiral EFT with (without) explicit $\Delta$-resonances. The dashed (solid) lines depict LO $(\mathrm{LO}+\mathrm{NLO})$ results.

tute the first systematic corrections to the leading $2 \pi$ exchange that appears at $\mathrm{N}^{2} \mathrm{LO}$. Another two contributions are of shorter range and include, additionally to an exchange of pions, also one short-range contact interaction and all corresponding $1 / m$ corrections. The requirement of renormalizability leads to unique expressions for $\mathrm{N}^{3} \mathrm{LO}$ contributions to the three-nucleon force (except for $1 / \mathrm{m}$ corrections).

We presented the complete $\mathrm{N}^{2} \mathrm{LO}$ analysis of the nuclear forces with explicit $\Delta$-isobar degrees of freedom. Although the overall results in the isospin-conserving case are very similar in the $\Delta$-less and $\Delta$-full theories we found a much better convergence in all peripheral partial waves once $\Delta$-resonance is explicitely taken into account. The leading CSB contributions to nuclear forces are proportional to nucleon- and $\Delta$-mass splittings. There appear strong cancellations between the two contributions which at leading order yield weaker $V^{I I I}$ potentials. This effect is, however, entirely compensated at subleading order such that the results in the theories with and without explicit $\Delta$ degrees of freedom are remarkably similar.

We are looking forward to numerical studies of $\mathrm{N}^{3} \mathrm{LO}$ three-nucleon forces and implementations of $\mathrm{N}^{2} \mathrm{LO}$ forces in the $\Delta$-full EFT in future NN partial-wave analyses.

\section{References}

1. H. Yukawa, Proc. Phys. Math. Soc. Jap. 17, 48 (1935).

2. S. Weinberg, Physica A 96, 327 (1979).

3. J. Gasser and H. Leutwyler, Annals Phys. 158, 142 (1984).

4. V. Bernard, Prog. Part. Nucl. Phys. 60, 82 (2008) [arXiv:0706.0312 [hep-ph]].

5. S. Weinberg, Nucl. Phys. B 363, 3 (1991).

6. V. G. J. Stoks, R. A. M. Kompl, M. C. M. Rentmeester and J. J. de Swart, Phys. Rev. C 48, 792 (1993).

7. NN-Online program, M. C. M. Rentmeester et al., http://nn-online.org.

8. SAID on-line program, R. A. Arndt et al., http://gwdac.phys.gwu.edu. [9]

9. E. Epelbaum, H. W. Hammer and U.-G. Meißner, arXiv:0811.1338 [nucl-th]. Rev. Mod. Phys., in print.

10. E. Epelbaum, Prog. Part. Nucl. Phys. 57, 654 (2006) [arXiv:nucl-th/0509032].

11. E. Epelbaum and U.- G. Meißner, Phys. Rev. C 72, 044001 (2005) [arXiv:nucl-th/0502052].

12. S. Ishikawa and M. R. Robilotta, Phys. Rev. C 76, 014006 (2007) [arXiv:0704.0711 [nucl-th]].

13. V. Bernard, E. Epelbaum, H. Krebs and U.G. Meißner, Phys. Rev. C 77, 064004 (2008) [arXiv:0712.1967 [nucl-th]].

14. E. Epelbaum, Phys. Lett. B 639, 456 (2006) [arXiv:nucl-th/0511025].

15. T. R. Hemmert, B. R. Holstein and J. Kambor, J. Phys. G 24, 1831 (1998) [arXiv:hep-ph/9712496].

16. C. Ordonez, L. Ray and U. van Kolck, Phys. Rev. C 53, 2086 (1996) [arXiv:hep-ph/9511380].

17. N. Kaiser, S. Gerstendorfer and W. Weise, Nucl. Phys. A 637 (1998) 395 [arXiv:nucl-th/9802071].

18. H. Krebs, E. Epelbaum and U.-G. Meißner, Eur. Phys. J. A 32, 127 (2007) [arXiv:nucl-th/0703087].

19. J. Fujita and H. Miyazawa, Prog. Theor. Phys. 17 (1957) 360.

20. U.-G. Meißner, AIP Conf. Proc. 1011, 49 (2008). 
$19^{\text {th }}$ International IUPAP Conference on Few-Body Problems in Physics

21. E. Epelbaum, H. Krebs and U.-G. Meißner, Nucl. Phys. A 806, 65 (2008) [arXiv:0712.1969 [nucl-th]].

22. N. Fettes, U.-G. Meißner and S. Steininger, Nucl. Phys. A 640, 199 (1998) [arXiv:hep-ph/9803266].

23. E. Epelbaum, H. Krebs and U.-G. Meißner, Phys. Rev. C 77, 034006 (2008) [arXiv:0801.1299 [nucl-th]].

24. E. Epelbaum, H. Krebs and U.-G. Meißner, forthcoming.

25. H. R. Rubinstein, F. Scheck and R. H. Socolow, Phys. Rev. 154, 1608 (1967). 